\title{
The effect of "robust" integrator dynamics on decision-making performance
}

\author{
Nicholas Cain ${ }^{1 *}$, Andrea Barreiro ${ }^{1}$, Mike Shadlen$^{2}$, Eric Shea-Brown ${ }^{1}$ \\ From Nineteenth Annual Computational Neuroscience Meeting: CNS*2010 \\ San Antonio, TX, USA. 24-30 July 2010
}

The drift-diffusion model of decision-making provides a framework to explain performance statistics of twoalternative perceptual decision-making tasks. In addition, the cortical activity of rhesus monkeys performing these tasks has been observed to stochastically fluctuate until a threshold level of activity is reached, implying a neuronal implementation of this decision-making paradigm [1].

The observed ramping of activity is hypothesized to represent the accumulation of sensory evidence over timescales much longer than those of the underlying cortical hardware. One proposed mechanism to implement this short-term cortical memory is by a self-excitatory recurrent neural integrator [2]. However, it has been observed that naïve excitatory recurrent circuits require extremely fine-tuning to accurately integrate an input signal [3].

We investigated the consequences of implementing a short-term evidence accumulation circuit via a recurrent network designed to be robust to mistuning in the global self-excitation parameter [4], [5]. Following the approach of previous work, we derived a firing rate model for the dynamics of the integrator parameterized by biophysically relevant quantities. In particular, a single piecewise-defined ordinary differential equation models the average firing rate activity of a number of bistable sub-populations exhibiting hysteresis.

A major consequence of this robust integration scheme is the exclusion of instantaneous sensory evidence that does not sufficiently favor one alternative over the other. In particular, if stimulus is below a certain evidence-exclusion threshold, average integrator activity does not change. This evidence exclusion mechanism reveals a potential performance tradeoff

\footnotetext{
* Correspondence: nicain@amath.washington.edu

'Department of Applied Mathematics, University of Washington, Seattle, WA, 98195, USA
}

between gaining robustness to mistuning on one hand, but ignoring potentially relevant information on the other.

To our surprise, we find that a surprisingly large fraction of sensory evidence can be ignored before decisionmaking performance is compromised. To explain this observation, we derived equations that describe a hierarchy of sequential hypothesis tests that vary in their evidence-exclusion threshold. These models yield quantitative predictions of how decision-making performance is diminished as model robustness is increased.

We conclude that for a fixed task difficulty and a constant decision threshold, a substantial fraction of the stimulus can be ignored before error rates or reaction times increase, if decision boundaries are modified appropriately. This observation implies that an evidence-accumulating neural integrator circuit robust to feedback mistuning might be able to underlie perceptual decision-making, without a strong compromise in performance.

\section{Acknowledgements}

The authors thank Adrienne Fairhall, Michael Shadlen, and their respective lab groups, for numerous helpful discussions. The authors also acknowledge funding support from the Northwest Center for Neural Engineering, and a Burroughs-Wellcome Fund Career Award at the Scientific Interface (E.S.-B.).

\section{Author details}

'Department of Applied Mathematics, University of Washington, Seattle, WA, 98195, USA. ²Department of Physiology and Biophysics, University of Washington, Seattle, WA, 98195, USA.

Published: 20 July 2010

\section{References}

1. Roitman J, Shadlen M: Response of neurons in the lateral intraparietal area during a combined visual discrimination reaction time task. J Neurosci 2002, 22:9475-9489.

2. Wang XJ: Decision Making in Recurrent Neuronal Circuits. Neuron 2008, 60:215-234. 
3. Seung $S$, Lee D, Reis B, Tank D: Stability of the memory of eye position in a recurrent network of conductance-based model neurons. Neuron 2000, 26:259-271.

4. Koulakov A, Raghavachari S, Kepecs A, Lisman J: Model for a robust neural integrator. Nat Neurosci 2002, 5(8):775-782.

5. Goldman M: Robust Persistent Neural Activity in a Model Integrator with Multiple Hysteretic Dendrites per Neuron. Cereb Cortex 2003, 13(11):1185-1195.

doi:10.1186/1471-2202-11-S1-P97

Cite this article as: Cain et al:: The effect of "robust" integrator dynamics on decision-making performance. BMC Neuroscience 2010 11(Suppl 1):P97.

Submit your next manuscript to BioMed Central and take full advantage of:

- Convenient online submission

- Thorough peer review

- No space constraints or color figure charges

- Immediate publication on acceptance

- Inclusion in PubMed, CAS, Scopus and Google Scholar

- Research which is freely available for redistribution

Submit your manuscript at www.biomedcentral.com/submit 